\title{
Document Version Relationship
}

National Cancer Institute

\section{Source}

National Cancer Institute. Document Version Relationship. NCI Thesaurus. Code C93461.

Specifies the meaning (or semantics) of the relationship between one document version and another. 